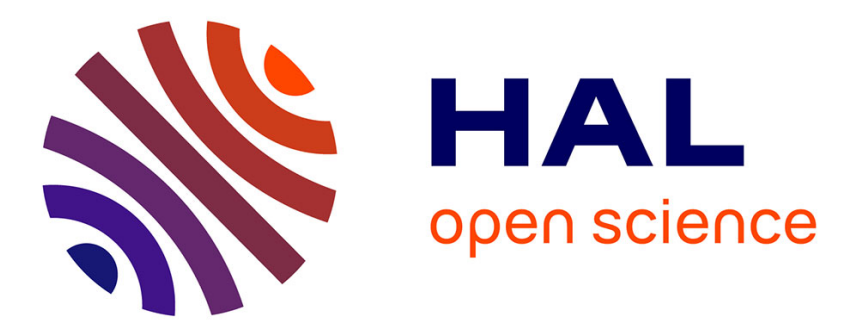

\title{
Use of topological defects as templates to direct assembly of colloidal particles at nematic interfaces
}

\author{
Mohamed Amine Gharbi, Maurizio Nobili, Christophe Blanc
}

\section{To cite this version:}

Mohamed Amine Gharbi, Maurizio Nobili, Christophe Blanc. Use of topological defects as templates to direct assembly of colloidal particles at nematic interfaces. Journal of Colloid and Interface Science, 2014, 417, pp.250-255. 10.1016/j.jcis.2013.11.051 . hal-00921311

\section{HAL Id: hal-00921311 \\ https://hal.science/hal-00921311}

Submitted on 22 Sep 2021

HAL is a multi-disciplinary open access archive for the deposit and dissemination of scientific research documents, whether they are published or not. The documents may come from teaching and research institutions in France or abroad, or from public or private research centers.
L'archive ouverte pluridisciplinaire HAL, est destinée au dépôt et à la diffusion de documents scientifiques de niveau recherche, publiés ou non, émanant des établissements d'enseignement et de recherche français ou étrangers, des laboratoires publics ou privés. 


\title{
Use of topological defects as templates to direct assembly of colloidal particles at nematic interfaces
}

\author{
Mohamed Amine Gharbi ${ }^{\mathrm{a}, \mathrm{b}, *}$, Maurizio Nobili ${ }^{\mathrm{a}}$, Christophe Blanc ${ }^{\mathrm{a}, *}$ \\ ${ }^{a}$ Laboratoire Charles Coulomb (L2C), UMR5221 CNRS and Université Montpellier 2, Place Eugène Bataillon, 34095 Montpellier, France \\ ${ }^{\mathrm{b}}$ Department of Physics and Astronomy, University of Pennsylvania, 209 S. 33rd Street, Philadelphia, PA 19104, USA
}

\begin{abstract}
In this work, we experimentally investigate the ability of topological defects to guide interfacial assembly of spherical particles with homeotropic anchoring confined to nematic interfaces. We propose two different systems: In the first one, particles are trapped at an air/nematic interface where they spontaneously form various 2D patterns. We demonstrate that the phase transition between these patterns can be controlled by defects formed in the nematic bulk. In the second system, we explore the behavior of particles at the surface of bipolar nematic drops. We found that particles assemble into linear chains and interact with surface defects at the North and South poles of the drop, giving rise to the formation of star structures in a self-assembly process. We detail the mechanism that guides the behavior of particles and discuss the role of defects in the formation of the observed patterns.
\end{abstract}

\section{Introduction}

During the past 30 years, there has been an increasing interest in investigating particles trapped at interfaces between two immiscible liquids [1-4]. It is known that the interfacial environment between isotropic phases causes a range of interparticle forces that guide the assembly of particles and arrange them into two-dimensional systems with rich phase properties [5-8].

Recently, it has become evident that the trapping of particles at complex fluid interfaces, nematic (N) liquid crystal (LC) interfaces for example, provides additional mechanisms to control 2D ordering transitions, with the ability of building complex artificial structures [9-16]. Let us recall that in a nematic liquid crystal, molecules are locally oriented on average in the same direction, the so-called director $n$. The presence of the director field strongly modifies the physics of nematic/isotropic liquid interfaces. First, the interfacial energy density $\gamma(n \cdot k)$ now depends on the direction of $n$ with respect to the interface normal $k$. It usually shows a minimum energy when the director is either parallel (planar anchoring) or perpendicular (homeotropic anchoring) to the interface, which tends to distort the director in the preferred direction. Second, a bulk free energy density is attached to the space variations of the director field $n$ [17] and the distortions due to the anchoring

* Corresponding authors. Address: Department of Physics and Astronomy, University of Pennsylvania, 209 S. 33rd Street, Philadelphia, PA 19104, USA (M.A. Gharbi).

E-mail addresses: mgharbi@sas.upenn.edu (M.A. Gharbi), christophe.blanc02@ univ-montp2.fr (C. Blanc). might be energetically costly. Even if generalized Young-Laplace equations (see for example [18]) can be found in the literature, the simplest capillary/elastic problems (such as finding the shape and director field of a nematic droplet) are technically cumbersome. However, fluids (such as air or water) having large interfacial energies (larger than $10^{-3} \mathrm{~J} \mathrm{~m}^{2}$ ) with common thermotropic liquid crystals are much simpler to describe since interface energy dominates nematic elastic energy and therefore controls alone the interface geometry. The director field is then constrained by the boundary conditions and arranges itself to minimize the elastic free energy (classic limit of strong anchoring conditions [19]). The same effects are responsible for the appearance of long-range elastic forces between trapped particles at the interfaces. Due to the nematic anchoring at their surface, particles modify the director in their vicinity. New structures emerge due to the interplay between elastic forces and other lateral interparticles forces that act parallel to the interface. For example we have recently shown that colloidal particles with homeotropic anchoring trapped at flat air/ nematic interface form various structures ranging from 1D chains to $2 \mathrm{D}$ patterns (stable liquid, hexagonal crystal and amorphous aggregates), depending on the anchoring conditions and the density of trapped particles [20]. In this first set of experiments, the liquid crystal was initially homogeneous and the patterns were not spatially controlled. Moreover transitions between the patterns could not be spontaneously obtained, due to the negligible capillary forces, which were dominated by the strong elastic repulsions at NLC interfaces [21,22]. The present work was stimulated by studies $[23,24]$ which have shown that colloidal particles dispersed in bulk NLC could also interact with fixed defects (disclinations) via 
elastic interactions. In bulk, particles are finally trapped at the core of the defects which provides an original template mechanism but we could expect that the simultaneous trapping by the interface could provide additional flexible strategies.

In this work, we therefore present an experimental study where we use defects, created in bulk or at nematic surface, to guide interfacial assembly of homeotropic silica beads. We offer two systems obtained by different methods. In the first one, we describe the behavior of particles at an air/nematic interface in the presence of defect lines in bulk. We then detail the role of those defects in confining particles at the interface and controlling their phase diagram. In the second system, we investigate the behavior of particles confined at complex nematic interfaces: at the surface of bipolar droplets. We thus discuss the role of surface defects (boojums) in the creation of new patterns with star-like symmetry.

\section{Materials and methods}

\subsection{Experimental systems}

The first system was obtained by trapping dry silica particles of diameter $2 R=3.92 \mu \mathrm{m}$ (from Bangslabs) at flat air/nematic interface following reference [20]. The nematic film (thickness range between 10 and $120 \mu \mathrm{m}$ ) was prepared by spin coating 4-pentyl4-cyanobiphenyl (5CB from Synthon) on a glass slide treated with polyvinyl alcohol (PVA from Sigma-Aldrich) and rubbed along one direction, to ensure oriented planar anchoring. The texture of the nematic is hybrid as shown in Fig. 1, due to the strong perpendicular anchoring of 5CB in contact with air (planar in contact with the base and perpendicular in contact with air).

The second system was obtained by trapping silica particles at the surface of bipolar 5CB droplets dispersed in aqueous solution that contains $1 \mathrm{wt} \%$ of PVA, generated in microfluidic glass capillary device following reference [25] (see Fig. 2). The PVA stabilizes $5 \mathrm{CB}$ droplets and ensures strong planar anchoring at the $5 \mathrm{CB} /$ water interface. Used silica particles were covered with a monolayer of N,N-dimethyl-N-octadecyl-3-aminopropyl trimethoxysilyl chloride (DMOAP purchased from Aldrich) to impose perpendicular anchoring of the nematic at their surfaces. All experiments were performed at room temperature $T=23^{\circ} \mathrm{C}$.

\subsection{Materials characterization}

We used a polarising microscope (LEICA DM 2500P) to observe studied systems equipped with an INSTEC hot stage, a SONY

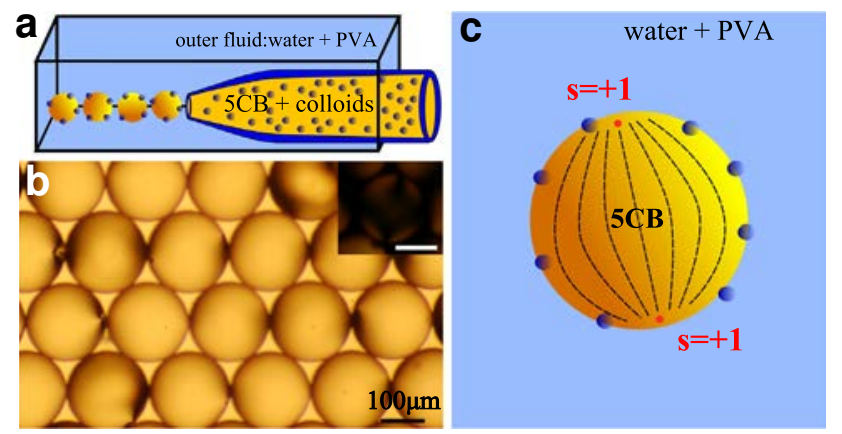

Fig. 2. (a and c) Experimental set-up used to generate monodisperse nematic droplets (b). Inset of (b): POM image of the bipolar configuration of the $5 \mathrm{CB}$ drop (bar scale is $10 \mu \mathrm{m}$ ). Silica particles are initially dispersed in the nematic liquid crystal. During the process of droplet formation, they are captured by the water/ nematic interface.

$1024 \times 768$ digital camera and a Nikon color camera. The shape of the air/nematic interface was characterized using home built interferometric techniques: Vertical Scanning Interferometry (VSI) and Phase Shift Interferometry (PSI). Optical tweezers based on a LEICA DMI 3000 B inverted microscope equipped with a $100 \times$ (NA 1.4) oil immersion objective, a $1064 \mathrm{~nm}$ laser (YLM $5 \mathrm{~W}$ from IPG Photonics) and a piezoelectric XY stage (MCL), were also used to manipulate individual particles.

\section{Results and discussions}

\subsection{Silica particles at an air/nematic interface}

We prepared hybrid nematic films of controlled thickness by imposing unidirectional planar anchoring of the $5 \mathrm{CB}$ in contact with the lower substrate. This configuration causes distorted alignment of the director that could involve topological defects, disclinations, separating two domains of different orientations as shown in Fig. 1(a) and (c). The nature of these disclinations evolves along a loop (Fig. 1(b)), from a disclination splay-bend of winding number $m=+1 / 2$ (Fig. 1(a)) to a disclination of winding number $m=-1 / 2$ (Fig. 1(c)), as illustrated in the sketch of Fig. 1(d) [26]). Experientially, these $\pm 1 / 2$ disclinations can be created by heating the nematic to the isotropic phase then cooling the system below the nematic-to-isotrope phase transition temperature $T_{N I}$. Typically, the loops spontaneously shrink after formation and decrease
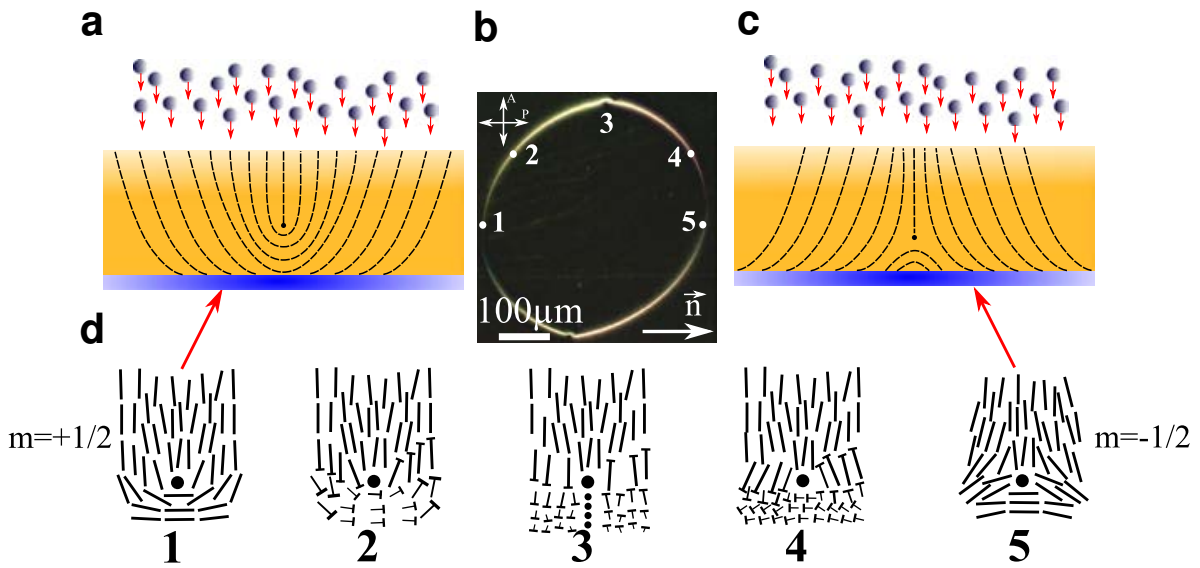

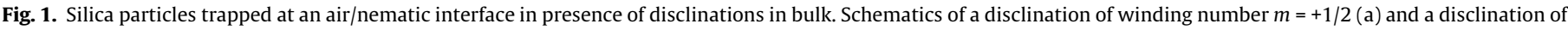

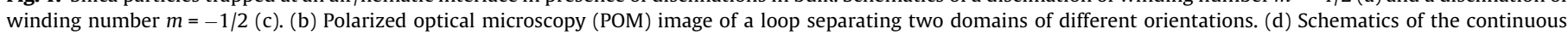
transformation along the loop from a disclination $m=+1 / 2$ to a disclination $m=-1 / 2$. 
their areas. Over time (few minutes), they vanish to minimize the excess free energy of the costly defects. However, these defects may be stabilized by different methods: by controlling boundary shapes [27-30], by dispersing colloidal particles in the nematic bulk $[23,24]$ or by trapping particles at nematic interfaces as showed in what follows. When homeotropic particles are trapped at air/nematic interfaces with hybrid texture, they form different patterns depending on the density of deposited particles and the boundary conditions of the system. The behavior of particles at hybrid films was reported in previous work [20]. In this study, we focus on the role of topological defects in controlling interfacial assembly of particles.

The silica beads are captured at the air/nematic interface due to their strong binding energy $\Delta E \sim 10^{7} k_{B} T$. The vertical position of trapped microspheres on a simple liquid would be simply controlled by the contact angle $\theta$, given by the Young equation which describes the balance of tension forces at the triple line. It writes: $\cos \theta=\left(\gamma_{p a}-\gamma_{p l c}\right) / \gamma_{a l c}$ where $\gamma_{p a}, \gamma_{p l c}$ and $\gamma_{\text {alc }}$ are the energies per unit area of particle/air interface, particle/liquid interface and air/liquid interface, respectively. With a nematic phase, the role of elasticity cannot be entirely dismissed even in case of strong anchoring conditions because a strong anchoring at both air and silica necessarily yields a defect at the contact line and therefore a diverging elastic energy density $[31,32]$. Following the analysis made by Rey [31] a homeotropic anchoring at both surfaces yields a modified Young equation: $\gamma_{a l c} \cos \theta-\left(\gamma_{p a}-\gamma_{p l c}\right)-K E^{2} \sin \theta / 2 r_{c}=0$. The additional term accounts for the nematic elastic energy with a bulk nematic modulus $K \approx 10^{-11} \mathrm{~N}$ for $5 \mathrm{CB}$ in the one constant approximation and $r_{c} \approx 10 \mathrm{~nm}$ a cut-off length near the defect. With the experimental value $\theta=33 \pm 2^{\circ}$ (see below), the constant $E=-\theta /(\pi-\theta) \approx-0.22$ [31]. The large $5 \mathrm{CB} /$ air interfacial energy $\gamma=20 \mathrm{mN} \mathrm{m}^{-1}$ is however sufficient for neglecting the last term since $\gamma_{\text {alc }} \cos \theta \approx 10^{3} K E^{2} \sin \theta / 2 r_{c}$. We employed the VSI technique to measure $\theta$. Typical reconstructions of the three-dimensional relief of an individual particle at the interface are given in Fig. 3(a) and (b). With VSI technique, we can detect the top of particles and their surrounding fluid (not their full 3D profiles), then deduce $\theta=33 \pm 2^{\circ}$ using the relation $\theta=\arccos (1-h / R)$, where $h$ is the height contrast and $R$ is the particle radius (Fig. 3(c)). Thus, particles appear narrower as shown in Fig. 3(a), due to the limited numerical aperture of the Mirau objective that we used. We validated interferometric results by measuring macroscopically the contact angle of $5 \mathrm{CB}$ droplets at silanized glass slides $\theta=33 \pm 3^{\circ}$ (Fig. 3(d)). Furthermore, we found that particles do not affect the shape of the interface, using the PSI technique with a resolution of the order of few nanometres (Fig. 3(e)). The angular distortions caused by particles are very small, less than $10^{-4}$ rad as shown in Fig. 3(f). Moreover, we were not able to measure the residual multipolar distortion that could be present even for microspheres due to the roughness of the particles and the pinning of the contact line [33]. This indicates that the dominant quadrupolar term is smaller than a few tens of nanometers at the contact line.

The beads at the nematic interface are very stable. They form various patterns similar to those described in reference [20]. For thin films, they assemble into linear chains parallel to the director orientation. When the thickness increases, they repel each other and form 2D stable liquid phase. In presence of disclinations, we observed a different colloidal behavior. For thin layers, thickness less than $25 \mu \mathrm{m}$, particles are very sensitive to the presence of bulk defects. They are spontaneously captured by them (Fig. 4(a)) and may form microscopic necklaces when the defects shrink over time (Fig. 4(b)). For intermediate thicknesses between 40 and $100 \mu \mathrm{m}$, the trapping effect is still strong and trapped particles form new types of necklaces with a typical distance separation around $10 \mu \mathrm{m}$ between each other (Fig. 4(c) and (d)). In both thin and
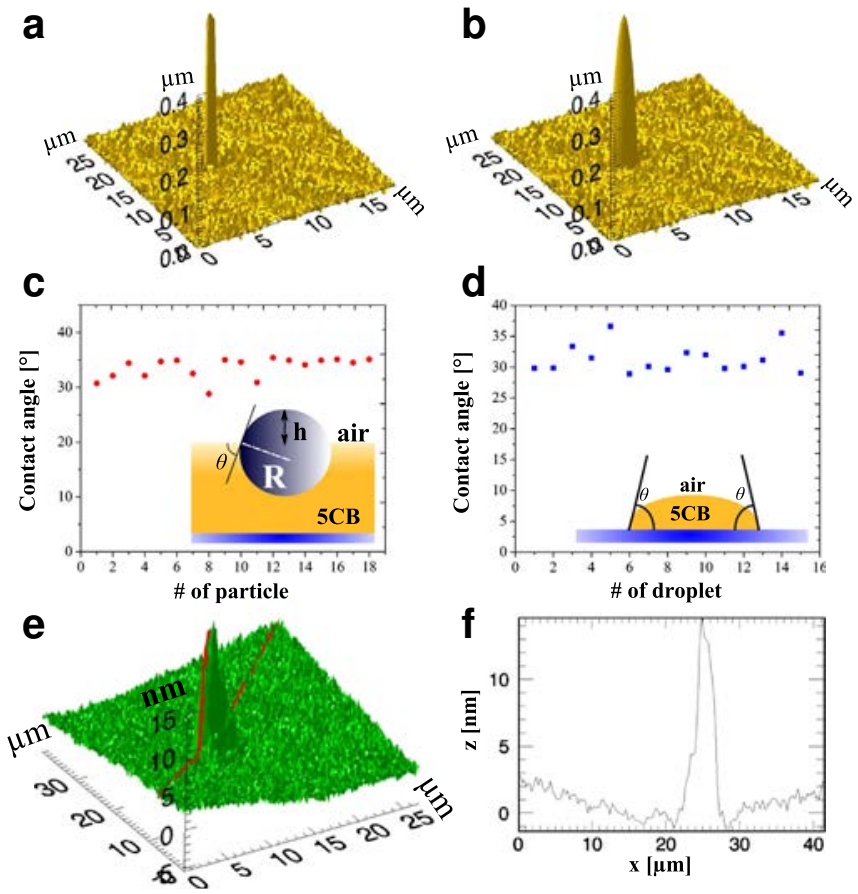

Fig. 3. (a) Typical reconstruction of the topographic profile of a particle with homeotropic anchoring trapped at an air/nematic interface using VSI technique. (b) The real profile of the spherical particle. (c) Microscopic measurements of the contact angle of particles with homeotropic anchoring at the air/nematic interface. (d) Macroscopic measurement of the contact angle of nematic droplets on glass slides treated with DMOAP. (e) Typical reconstruction of the interface topography around an isolated particle using PSI technique and its corresponding vertical distortion (f)

intermediate films, the particles stabilize disclinations and reduce their speed of shrinking. However, the interaction between particles and defects decreases when increasing the thickness of the nematic film. It vanishes above $100 \mu \mathrm{m}$, showing that elastic forces are limited by the thickness of the nematic film. The particles form then 2D patterns depending only on the conditions of confinement at the interface as shown in Fig. 4(e) and (f).

To probe interactions between like particles and those between disclinations and particles, we used the technique of optical tweezers. This technique allowed us to manipulate isolated beads as well as to establish their interactions. We found that particles are strongly attached to disclinations; it is very difficult to liberate them from the defect line even for high laser power. We also checked that confined particles repel each other at intermediate thickness, by approaching them closely to each other then tracking their positions after trap release (Fig. 5(a) and (b)). In addition to that, a crossover from repulsion to attraction was observed when two particles are approached too close to each other, which is reminiscent of the elastic attraction between particles at air/nematic interface described in Ref. [20]. Thus, we can build necklaces similar to those obtained at thin layers (Fig. 4(a)), just by forcing many particles to touch each other as shown in Fig. 5(c).

As said above, bulk defects can be created by heating the nematic to the isotropic phase, then cooling it back below the nematicto-isotropic phase transition temperature. After formation, these defects trap particles and shrink by confining their surrounding particles until they are stabilized. During this process, various patterns spontaneously form with different symmetry. We obtain then 2D amorphous aggregates decorating the defect line near large crystalline hexagonal domains. However, far away from the disclination, a stable 2D liquid phase is observed (Fig. 5(d)). All these patterns disappear when the nematic is heated to the 

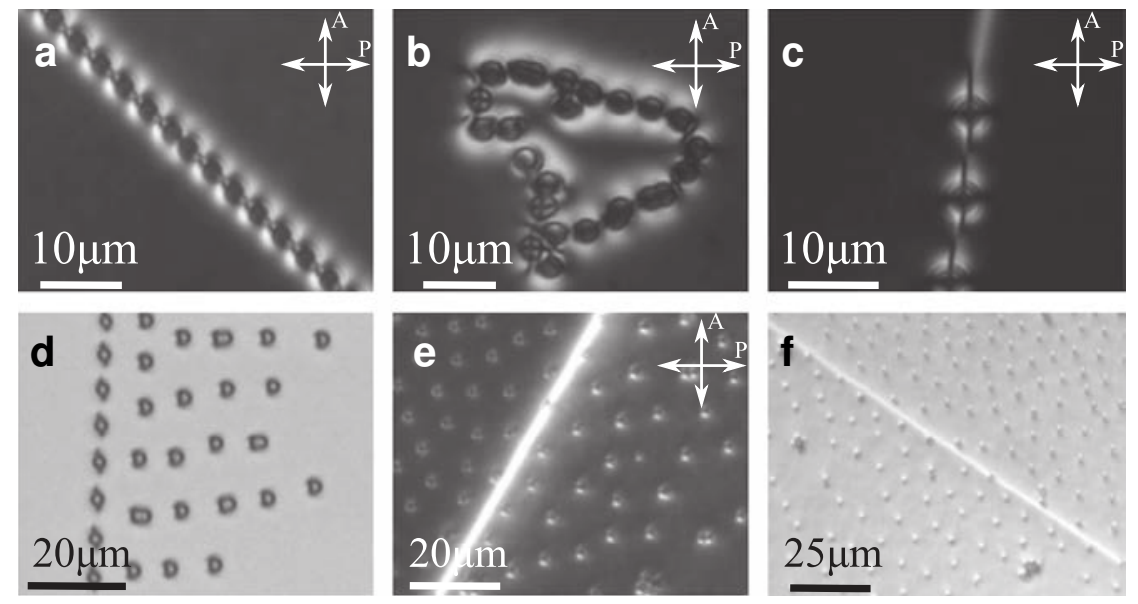

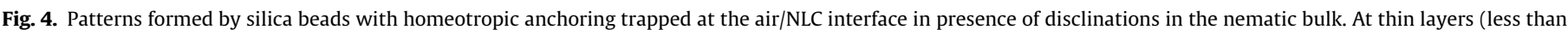

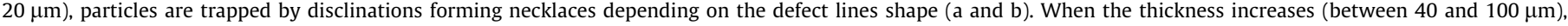

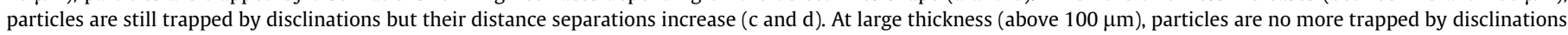
(e and f), patterns very sensitive to the conditions of confinement are then observed.
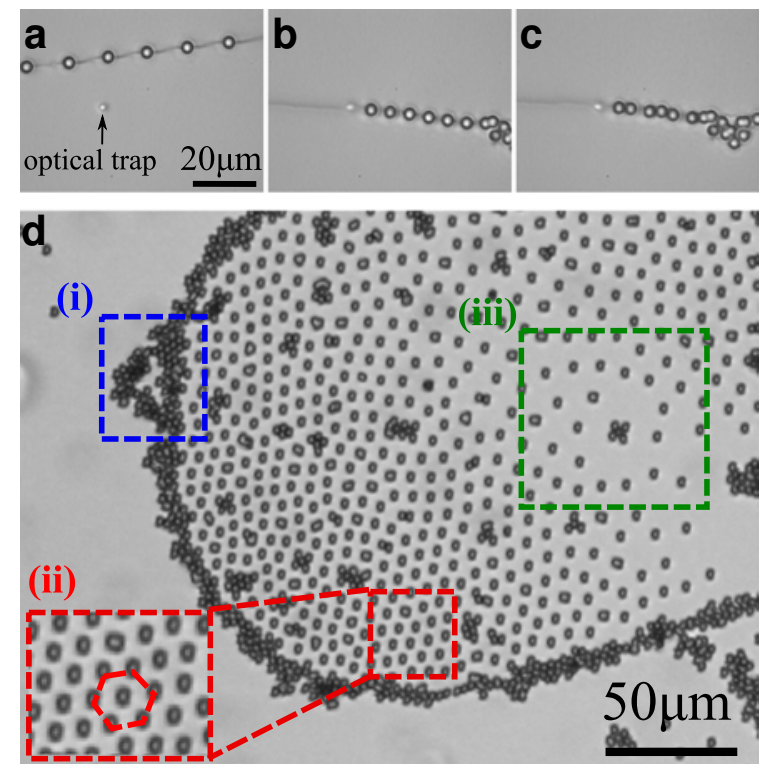

Fig. 5. (a-c) Use of optical tweezers to probe interactions between defects and particles as well as interactions between like particles at intermediate thicknesses $(\sim 80 \mu \mathrm{m})$. (a) Particles are strongly trapped by disclinations. They repeal each other (b). (c) When particles are approached too close to each other, a crossover from repulsion to attraction is observed and necklaces similar to those observed at thin films form. (d) 2D confinement of particles at the air/nematic interface and control of their phase behavior using a bulk defect. Close to the disclination, amorphous aggregates decorating the defect line (i) and large crystalline hexagonal domains are present (ii). Far away, the particles form stable liquid phases (iii).

isotropic phase, confirming that the elasticity is at the origin of particle organizations.

Due to the strong homeotropic anchoring of the $5 \mathrm{CB}$ at their surfaces, particles induce distortion of the director field when they are captured at the air/nematic interface. In addition to that, a defect associated with the colloid, that could be either a point defect (hedgehog hyperbolic) or a line defect (Saturn Ring) form in order to satisfy anchoring conditions at particle surface and at air/nematic interface. The structure of the director $n$ around the beads evolves depending on the thickness of the nematic film. It could be determined by minimizing the elastic free energy of the nematic/particle system $\mathcal{F}=K / 2 \int_{v} d^{3} r(\nabla n)^{2}$, by analogy with electrostatic [34]. The Euler-Lagrange equation arising from the minimisation of the elastic free energy reduces to the Laplace equation for $n$. At large distance the director is given by a multipole expansion in $n$. To leading order, the dipolar configuration gives $n^{i}=\operatorname{Pr}^{i} / r^{3}$ and the quadrupolar configurations gives $n^{i}=Q_{j k}^{i} r_{j} r_{k} / r^{5}, i=x, y$, where $P$ and $Q$ are the elastic dipole and quadrupole moments of the particle and its associated defect, respectively. A summation over $j$ and $k=x, y, z$ is implied. In the other hand, the hybrid texture of the nematic film creates disclinations in bulk with splay-bend distortion. To minimize deformations of $n$ and reduce the total elastic free energy $\mathcal{F}$ of the system, the splay-bend distortion created by bulk defects interact with the dipolar or the quadrupolar distortions created by particles. Thus, the defects attract beads via long-range elastic interactions and may build different types of necklaces with them, depending on if particles attract each other (at thin films, via dipolar elastic attraction $\propto K R^{4} / r^{3}$ ) or repeal each other (at intermediate films, via quadrupolar elastic repulsion $\left.\propto K R^{6} / r^{5}\right)$. These disclinations may also be used as templates to confine colloidal particles and control their 2D phase behavior in a self-assembly mechanism as shown in Fig. 5(d). The interactions between defects and particles act at large distance, however, they are limited by the extension of the director distortions (created by both particles and disclinations). For this reason they vanish at large films thicker than $100 \mu \mathrm{m}$. Finally, we conclude that bulk defects may spontaneously generate ordered structures at liquid crystal interfaces, which in turn could be considered as a new mechanism to guide interfacial assembly of colloidal particles.

\subsection{Particles confined at bipolar nematic droplets}

The second system is obtained by trapping silica particles with homeotropic anchoring at a water/nematic interface, where topological defects are created at precise locations. The motivation of this study is to investigate the role of surface defects in the assembly of particles at complex nematic interfaces. In many previous works, it has been shown that the confinement of a nematic liquid crystal to spherical geometries leads to a variety of director field configurations. These configurations depend on the boundary conditions imposed at the droplet surface (the anchoring for $n$ ) $[35,36]$. In our system, we generate monodisperse nematic droplets (of diameter between 150 and $250 \mu \mathrm{m}$ ) using micro-capillary devices. A strong tangential boundary conditions for $n$ is imposed at the water/nematic interface using an outer fluid that contains 
PVA. Under these conditions, two surface defects of topological charge $s=+1$ known as boojums form at the North and South poles of the drop. As a result, the drop presents bipolar configurations with total charge equal to $s_{\text {total }}=+2$, as required by the Poincaré-Hopf theorem [37,38](Fig. 2).

The silica beads are stable at the water/nematic interface. They are confined with a contact angle $\theta=37 \pm 2^{\circ}$, obtained by macroscopic measurements of the contact angle of $5 \mathrm{CB}$ droplets deposited on silanized glass and covered by a $1 \mathrm{wt} \%$ PVA aqueous solution. To satisfy boundary conditions, particles create distortion of the director field and induce the formation of point defects close to particles, the hyperbolic hedgehogs (inset of Fig. 6(a)), suggesting that the distortion is dipolar at large distance, by analogy with previous works in bulk [39,40], or at the interface [16,25]. Particles interact then with each other via dipolar elastic interactions and assemble into linear chains along longitude lines connecting the North and South poles of the 5CB drops as shown in Fig. 6(a) and (b) (along the director field orientation). These interactions are of elastic origin, because when the nematic is heated to the isotropic phase they vanish and chains are no more observed. The latter result also rules out the possibility of capillary interactions between particles and confirms that they are negligible at water/nematic interfaces, similar to the case of air/nematic interface showed in the previous section. The chains grow over time and migrate to the North and South poles of the drop where defects are located. After few hours, they spontaneously form star patterns around the boojums that disappear in the isotropic phase (Fig. 6(c) and (d)). Individual particles converge then to the bottom of the drop where they aggregate due to their density, which is higher than the densities of 5CB and water + PVA.

We conclude that the formation of star patterns at the surface of nematic droplets is a result of the collaboration between two colloidal interactions: (i) The first one is the dipole-dipole interaction between like particles that creates linear chains along the longitudinal orientation of the director field. (ii) The second one is an interaction between surface defects and chains that organizes them into star patterns. Both of them are of elastic origin, they vanish in the isotropic phase. We note finally that the designing of topological defects at the nematic surface by confining the liquid crystal to spherical geometries and under controlled boundary conditions, may be used as a mechanism to guide spontaneously the assembly of particles at water/nematic interfaces.

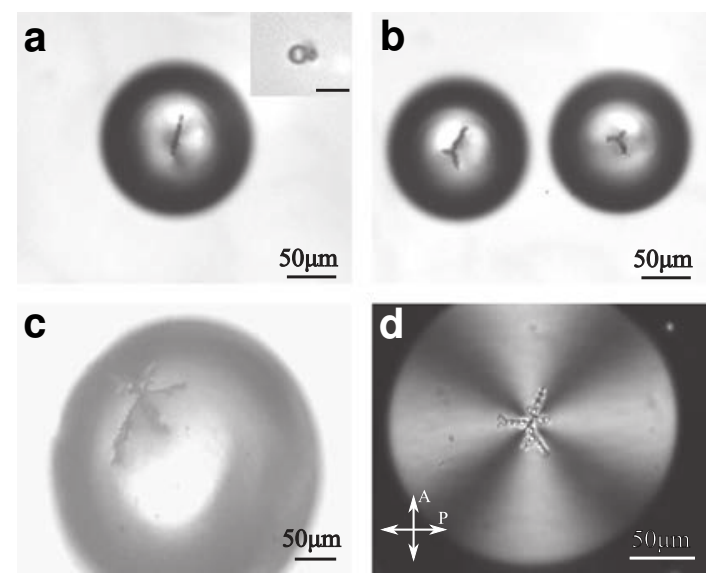

Fig. 6. Assembly of particles with homeotropic anchoring at the surface of bipolar nematic droplets. (a) The silica beads create elastic dipoles at the water/nematic interface and form linear chains along longitude lines parallel to the director field. Inset: a particle with its associated hyperbolic hedgehog (bar scale is $10 \mu \mathrm{m})$. (b-d) The linear chains interact with surface defects (the boojums) and assemble into star patterns around them.

\section{Conclusion}

The use of elastic distortions to guide the assembly of colloidal particles at liquid crystal interfaces is remarkably efficient. Recent advances in the field show an exceptional promise for significant new discoveries [25,41-43]. In this work, we demonstrate the ability of topological defects to control the 2D phase diagram of colloidal particles at a flat air/nematic interface and to organize particles into new star-like structures at a spherical water/nematic interface. By exploiting the mechanisms described in the paper, a variety of reconfigurable structures can be developed. In addition, we have showed that capillary forces between micro-particles confined to nematic interfaces are negligible in both cases. Particles interact with each other via dipole-dipole or quadrupole-quadrupole elastic interactions, which compete with other long-range interactions induced by defects that could be either in the nematic bulk or at the nematic surface. We have exhaustively described how these disclinations could be considered as templates to arrange particles at liquid crystal interfaces. The study of these materials could offer important insights and technological advancement for applications, such as photonics [44], Pickering emulsions [45], synthetic materials with tailored properties [46], and permeability control [47].

\section{Note}

We note that during the reviewing process of this work, an abstract of a study focused on the behaviour of particles at the surface of nematic droplets was published in Physical Review Letters website [48].

\section{Acknowledgments}

This work was supported in part by French ANR Grant BLAN07-1-183526 "Surfoids". M.A.G. acknowledges R. Gupta and V. Garbin for helpful discussions as well as MRSEC DMR11-20901 GRANTS.

\section{References}

[1] P. Pieranski, Phys. Rev. Lett. 45 (1980) 569-572.

[2] M. Oettel, A. Dominguez, S. Dietrich, Phys. Rev. E 71 (2005) 051401.

[3] R. McGorty, J. Fung, D. Kaz, V.N. Manoharan, Mater. Today 13 (2010) 34-42.

[4] L. Botto, E.P. Lewandowski, M. Cavallaro, K.J. Stebe, Soft Matter 8 (2012) 9957 9971.

[5] T. Terao, T. Nakayama, Phys. Rev. E 60 (1999) 7157.

[6] J. Loudet, B. Pouligny, Eur. Phys. J. E 34 (2011) 1-17.

[7] U. Gasser, C. Eisenmann, G. Maret, P. Keim, ChemPhysChem 11 (2010) $963-$ 970.

[8] J.-C. Loudet, A.M. Alsayed, J. Zhang, A.G. Yodh, Phys. Rev. Lett. 94 (2005) 018301.

[9] V. Nazarenko, A. Nych, B. Lev, Phys. Rev. Lett. 87 (2001). 075504-075504.

[10] D. Andrienko, M. Tasinkevych, P. Patricio, M.T. da Gama, Phys. Rev. E 69 (2004) 021706.

[11] I. Smalyukh et al., Phys. Rev. Lett. 93 (2004) 117801.

[12] A. Nych et al., Phys. Rev. Lett. 98 (2007) 057801.

[13] I.-H. Lin, G.M. Koenig Jr., J.J. de Pablo, N.L. Abbott, J. Phys. Chem. B 112 (2008) $16552-16558$.

[14] B. Lev, S. Chernyshuk, T. Yamamoto, J. Yamamoto, H. Yokoyama, Phys. Rev. E 78 (2008) 020701.

[15] T. Yamamoto, M. Yoshida, Appl. Phys. Express 2 (2009) 1501.

[16] G.M. Koenig, I.-H. Lin, N.L. Abbott, Proc. Nat. Acad. Sci. 107 (2010) 3998-4003.

[17] P.G. De Gennes, J. Prost, Int. Ser. Monogr. Phys. (2008) 83.

[18] A.D. Rey, J. Chem. Phys. 113 (2000) 10820-10822.

[19] P.S. Drzaic, Liquid Crystal Dispersions, World Scientific, Singapore, 1995.

[20] M.A. Gharbi et al., Soft Matter 7 (2011) 1467-1471.

[21] M. Oettel, A. Dominguez, M. Tasinkevych, S. Dietrich, Eur. Phys. J. E 28 (2009) 99-111.

[22] V. Pergamenshchik, Phys. Rev. E 79 (2009) 011407.

[23] D. Pires, J.-B. Fleury, Y. Galerne, Phys. Rev. Lett. 98 (2007) 247801.

[24] J.-B. Fleury, D. Pires, Y. Galerne, Phys. Rev. Lett. 103 (2009) 267801.

[25] M.A. Gharbi et al., Soft Matter 9 (2013) 6911-6920.

[26] M. Kléman, O.D. Laverntovich, Soft Matter Physics: An Introduction, Springer (2003). 
[27] T. Ohzono, J.-i. Fukuda, K. Suzuki, T. Yamaguchi, Phys. Rev. E 86 (2012) 030701.

[28] T. Ohzono, J.-i. Fukuda, Nat. Commun. 3 (2012) 701.

[29] G. Carbone, G. Lombardo, R. Barberi, I. Muševič, U. Tkalec, Phys. Rev. Lett. 103 (2009) 167801.

[30] B. Wang, R. Yamaguchi, M. Ye, S. Sato, Jpn. J. Appl. Phys. 41 (2002) 5307-5310.

[31] A.D. Rey, Langmuir 19 (2003) 3677-3685.

[32] R.H. Otten, P. van der Schoot, Langmuir 25 (2009) 2427-2436.

[33] D. Stamou, C. Duschl, D. Johannsmann, Phys. Rev. E 62 (2000) 5263.

[34] T. Lubensky, D. Pettey, N. Currier, H. Stark, Phys. Rev. E 57 (1998) 610.

[35] A. Fernández-Nieves, D. Link, M. Marquez, D.A. Weitz, Phys. Rev. Lett. 98 (2007) 087801.

[36] T. Lopez-Leon, A. Fernández-Nieves, Colloid Polym. Sci. 289 (2011) 345-359.

[37] N.D. Mermin, Rev. Mod. Phys. 51 (1979) 591.

[38] R.D. Kamien, Rev. Mod. Phys. 74 (2002) 953-971.
[39] P. Poulin, H. Stark, T. Lubensky, D. Weitz, Science 275 (1997) 1770-1773.

[40] P. Poulin, D. Weitz, Phys. Rev. E 57 (1998) 626.

[41] M. Cavallaro et al., Proc. Nat. Acad. Sci. (2013). http://dx.doi.org/10.1073/ pnas.1313551110.

[42] J.S. Lintuvuori et al., Phys. Rev. Lett. 110 (2013) 187801.

[43] F. Mondiot, X. Wang, J.J. de Pablo, N.L. Abbott, J. Am. Chem. Soc. 135 (2013) 9972-9975.

[44] H. Cong, B. Yu, J. Tang, Z. Li, X. Liu, Chem. Soc. Rev. 42 (2013) $7774-$ 7800.

[45] J.W. de Folter et al., Langmuir (2013). http://dx.doi.org/10.1021/la402427.

[46] Y. Yang, L. Gao, G.P. Lopez, B.B. Yellen, ACS Nano 7 (2013) 2705-2716.

[47] F. Yan, W.A. Goedel, Chem. Mater. 16 (2004) 1622-1626.

[48] J.K. Whitmer et al., Phys. Rev. Lett. (2013). http://prl.aps.org/accepted/ d707cY8fQ211ab43f71b81025c42fc94fd5def4c1. 